\title{
A general stability for a von Kármán system with memory
}

\section{Jum-Ran Kang*}

\section{"Correspondence:}

pointegg@hanmail.net

Department of Mathematics,

Dong-A University, Busan, 604-714, Korea

\begin{abstract}
In this paper we study the von Kármán plate model with long-range memory. We prove an explicit and general decay rate result using some properties of the convex functions. Our result is obtained without imposing any restrictive assumptions on the behavior of the relaxation function at infinity. These general decay estimates extend and improve on some earlier results: exponential or polynomial decay rates.
\end{abstract}

Keywords: von Kármán system; general decay rate; convexity; viscoelastic

\section{Introduction}

This paper is concerned with the general decay of the solutions to a von Kármán system for the plate equation with memory:

$$
\begin{aligned}
& u^{\prime \prime}-h \Delta u^{\prime \prime}+\Delta^{2} u-\int_{0}^{t} g(t-s) \Delta^{2} u(s) d s=[u, v] \quad \text { in } \Omega \times(0, \infty), \\
& \Delta^{2} v=-[u, u] \quad \text { in } \Omega \times(0, \infty), \\
& v=\frac{\partial v}{\partial v}=0 \quad \text { on } \Gamma \times(0, \infty), \\
& u=\frac{\partial u}{\partial v}=0 \quad \text { on } \Gamma_{0} \times(0, \infty), \\
& \mathcal{B}_{1} u-\mathcal{B}_{1}\left\{\int_{0}^{t} g(t-s) u(s) d s\right\}=0 \quad \text { on } \Gamma_{1} \times(0, \infty), \\
& \mathcal{B}_{2} u-h \frac{\partial u^{\prime \prime}}{\partial v}-\mathcal{B}_{2}\left\{\int_{0}^{t} g(t-s) u(s) d s\right\}=0 \quad \text { on } \Gamma_{1} \times(0, \infty), \\
& u(x, y, 0)=u_{0}(x, y), \quad u^{\prime}(x, y, 0)=u_{1}(x, y) \quad \text { in } \Omega,
\end{aligned}
$$

where $\Omega$ is an open bounded set of $\mathbb{R}^{2}$ with a sufficiently smooth boundary $\Gamma=\Gamma_{0} \cup \Gamma_{1}$. Here, $\Gamma_{0}$ and $\Gamma_{1}$ are closed and disjoint. The equations describe small vibrations of a thin homogeneous isotropic plate of uniform thickness $h$. Let us denote by $v=\left(v_{1}, v_{2}\right)$ the external unit normal to $\Gamma$, and by $\eta=\left(-v_{2}, v_{1}\right)$ the unitary tangent positively oriented on $\Gamma$. Here

$$
\mathcal{B}_{1} u=\Delta u+(1-\mu) B_{1} u \quad \text { and } \quad \mathcal{B}_{2} u=\frac{\partial \Delta u}{\partial v}+(1-\mu) B_{2} u,
$$

(c) 2015 Kang. This article is distributed under the terms of the Creative Commons Attribution 4.0 International License (http://creativecommons.org/licenses/by/4.0/), which permits unrestricted use, distribution, and reproduction in any medium, provided you give appropriate credit to the original author(s) and the source, provide a link to the Creative Commons license, and indicate if changes were made. 
where constant $\mu\left(0<\mu<\frac{1}{2}\right)$ is Poisson's ratio and

$$
\begin{aligned}
& B_{1} u=2 v_{1} v_{2} u_{x y}-v_{1}^{2} u_{y y}-v_{2}^{2} u_{x x}, \\
& B_{2} u=\frac{\partial}{\partial \eta}\left[\left(v_{1}^{2}-v_{2}^{2}\right) u_{x y}+v_{1} v_{2}\left(u_{y y}-u_{x x}\right)\right] .
\end{aligned}
$$

The von Kármán bracket is given by

$$
[u, v]=u_{x x} v_{y y}-2 u_{x y} v_{x y}+u_{y y} v_{x x}
$$

For the last several decades, the mathematical models of vibrating, flexible structures have been considerably stimulated by an increasing number of questions of practical concern. The main purpose of this monograph is to present a systematic study of uniform stabilization of the motion of a thin plate through the action of forces and moments applied at the edge of the plate. Among the elastic plate models, the von Kármán model is a 'large deflection' plate model, in a sense of a nonlinear analogue of the Kirchhoff model. However, it is assumed that the vertical deflection is small in comparison with the lateral dimensions of the plate. This hypothesis leads to a coupled pair of fourth-order, nonlinear partial differential equations for the vertical displacement $u$ and the Airy-stress function $v$. We may interpret Eq. (1.1) as saying that the stresses at any instant depend on the complete history of strains which the material has undergone. We will give later the precise condition on $g$ in order to obtain the general decay results.

The problem of stability of the solutions to a von Kármán system with dissipative effects has been studied by several authors. In [1-4] the authors considered the von Kármán system with frictional dissipations effective in the boundary. It is shown in these works that these dissipations produce uniform rate of decay of the solution when $t$ goes to infinity. Rivera and Menzala [5] and Rivera et al. [6] studied the stability of the solutions to a von Kármán system for viscoelastic plates with memory and boundary memory conditions, respectively. They proved that the energy decays uniformly exponentially or algebraically with the same rate of decay as the relaxation function. Later, Santos and Soufyane [7] generalized the decay result of [6]. Raposo and Santos [8] investigated the general decay of the solutions to a von Kármán plate model. Recently, Kang [9] proved the general decay of the solutions to a von Kármán plate model with memory and boundary damping. Kang [9] improved the results of [8] without imposing any restrictive growth assumption on the damping term and strongly weakening the usual assumption on the relaxation function.

On the other hand, the problem of stability of the solutions to a viscoelastic system with memory has been studied by many authors. In [10-13] the authors showed exponential and polynomial decay for a viscoelastic wave equation under the usual condition

$$
-c_{1} g(t) \leq g^{\prime}(t) \leq-c_{2} g(t) \quad \text { and } \quad 0 \leq g^{\prime \prime}(t) \leq c_{3} g(t)
$$

for some $c_{i}, i=1,2,3$. Later, this assumption was relaxed by several authors. Berrimi and Messaoudi [14] studied exponential and polynomial decay rates under condition on $g$ such as

$$
g^{\prime}(t) \leq-\xi g^{p}(t) \quad \text { for } 1 \leq p<\frac{3}{2}, t \geq 0
$$


where $\xi>0$. Messaoudi and Tatar [15] and Liu [16] considered exponential and polynomial decay for a quasilinear equation and a system of two coupled quasilinear viscoelastic equations under condition (1.8) by choosing a suitable perturbed energy, respectively. Messaoudi [17] and Han and Wang [18] proved a general decay rate for viscoelastic equations under a more general condition on $g$ such as

$$
g^{\prime}(t) \leq-\xi(t) g(t), \quad \frac{\left|\xi^{\prime}(t)\right|}{|\xi(t)|} \leq k, \quad \xi(t)>0, \xi^{\prime}(t) \leq 0, \forall t>0
$$

Guesmia and Messaoudi [19] obtained general stability for a Timoshenko system under weaker condition

$$
g^{\prime}(t) \leq-\xi(t) g(t)
$$

where $\xi$ is a nonincreasing and positive function. The stability of the solutions to a viscoelastic system under condition (1.10) was studied in [20-23] and the references therein. Mustafa and Messaoudi $[24,25]$ investigated the general stability result for a viscoelastic equation for a relaxation function satisfying

$$
g^{\prime}(t) \leq-H(g(t))
$$

where $H$ is a nonnegative function, with $H(0)=0$, and $H$ is a linear or strictly increasing and strictly convex on $(0, r]$ for some $r>0$. The above conditions are weaker conditions on $H$ than those imposed in [26]. Recently, Cavalcanti et al. [27] proved the uniform decay rates of the energy for solutions of a von Kármán system with long memory for the memory kernel $g$ satisfying (1.11).

Motivated by the work in [24-27], we establish an explicit and general decay of the solutions to a von Kármán plate model (1.1)-(1.7) for relaxation functions satisfying (1.11). The proof is based on the multiplier method and makes use of some properties of convex functions. This result improves on earlier ones in the literature because it allows certain relaxation functions which are not necessarily of exponential or polynomial decay.

The paper is organized as follows. In Section 2, we present some notations and material needed for our work and state a global existence theorem. In Section 3, we prove the general decay of the solutions to the von Kármán system with memory.

\section{Preliminaries}

In this section, we present some material needed in the proof of our result and state the main result. Throughout this paper we denote $(u, v)=\int_{\Omega} u(x, y) v(x, y) d \Omega$ and define

$$
V=\left\{v \in H^{1}(\Omega) \mid v=0 \text { on } \Gamma_{0}\right\}, \quad U=\left\{u \in H^{2}(\Omega) \mid u=\frac{\partial u}{\partial v}=0 \text { on } \Gamma_{0}\right\} .
$$

For a Banach space $X,\|\cdot\|_{X}$ denotes the norm of $X$. For simplicity, we denote $\|\cdot\|_{L^{2}(\Omega)}$ by $\|\cdot\|$.

A simple calculation, based on the integration by parts formula, yields

$$
\left(\Delta^{2} u, v\right)=a(u, v)+\left(\mathcal{B}_{2} u, v\right)_{\Gamma}-\left(\mathcal{B}_{1} u, \frac{\partial v}{\partial v}\right)_{\Gamma}
$$


where the bilinear symmetric form $a(u, v)$ is given by

$$
a(u, v)=\int_{\Omega}\left\{u_{x x} v_{x x}+u_{y y} v_{y y}+\mu\left(u_{x x} v_{y y}+u_{y y} v_{x x}\right)+2(1-\mu) u_{x y} v_{x y}\right\} d \Omega,
$$

where $d \Omega=d x d y$. Since $\Gamma_{0} \neq \emptyset$, we know that $\sqrt{a(u, u)}$ is equivalent to the $H^{2}(\Omega)$ norm on $U$, i.e.,

$$
c_{0}\|u\|_{H^{2}(\Omega)}^{2} \leq a(u, u) \leq \tilde{c}_{0}\|u\|_{H^{2}(\Omega)}^{2},
$$

where $c_{0}$ and $\tilde{c}_{0}$ are generic positive constants. This and the Sobolev imbedding theorem imply that for some positive constants $C_{p}$ and $C_{s}$,

$$
\|u\|^{2} \leq C_{p} a(u, u), \quad\|\nabla u\|^{2} \leq C_{s} a(u, u), \quad \forall u \in U .
$$

We consider the following hypotheses:

(H1) $g: \mathbb{R}^{+} \rightarrow \mathbb{R}^{+}$is a differentiable function such that

$$
g(0)>0, \quad l:=\int_{0}^{\infty} g(s) d s<1 .
$$

(H2) There exists a positive function $H \in C^{1}\left(\mathbb{R}^{+}\right)$, with $H(0)=0$, and $H$ is a linear or strictly increasing and strictly convex $C^{2}$ function on $(0, r]$ for some $r<1$ such that

$$
g^{\prime}(t) \leq-H(g(t)), \quad \forall t \geq 0
$$

To simplify calculation in our analysis, we introduce the following notation:

$$
\begin{aligned}
& (g * u)(t):=\int_{0}^{t} g(t-s) u(s) d s, \\
& (g \square u)(t):=\int_{0}^{t} g(t-s)\|u(\cdot, t)-u(\cdot, s)\|^{2} d s, \\
& \left(g \square \partial^{2} u\right)(t):=\int_{0}^{t} g(t-s) a(u(\cdot, t)-u(\cdot, s), u(\cdot, t)-u(\cdot, s)) d s .
\end{aligned}
$$

From the symmetry of $a(\cdot, \cdot)$ we have that for any $v \in C^{1}\left(0, T ; H^{2}(\Omega)\right)$,

$$
\begin{aligned}
a\left(g * v, v^{\prime}\right)= & -\frac{1}{2} g(t) a(v, v)+\frac{1}{2} g^{\prime} \square \partial^{2} v \\
& -\frac{1}{2} \frac{d}{d t}\left\{g \square \partial^{2} v-\left(\int_{0}^{t} g(s) d s\right) a(v, v)\right\} .
\end{aligned}
$$

We introduce the following lemma for the bracket's binary.

Lemma 2.1 ([28]) Let $u, w \in H^{2}(\Omega)$ and $v \in H_{0}^{2}(\Omega)$, where $\Omega$ is an open bounded and connected set of $\mathbb{R}^{2}$ with regular boundary. Then

$$
\int_{\Omega} w[v, u] d \Omega=\int_{\Omega} v[w, u] d \Omega
$$


Lemma 2.2 ([1]) If $u, v \in H^{2}(\Omega)$, then $[u, v] \in L^{2}(\Omega)$ and satisfies

$$
\|[u, v]\| \leq c_{0}^{\prime}\|u\|_{H^{2}}\|v\|_{W^{2, \infty}} \text { and }\|v\|_{W^{2, \infty}} \leq c_{0}^{\prime}\|u\|_{H^{2}}^{2} .
$$

By using Galerkin's approximation, we can obtain the following result for the solution. For the initial data $\left(u_{0}, u_{1}\right) \in H^{4}(\Omega) \times H^{2}(\Omega), h>0$, system (1.1)-(1.7) has a unique weak solution $u$ in the following class:

$$
u \in L^{\infty}\left(0, \infty ; U \cap H^{4}(\Omega)\right), \quad u^{\prime} \in L^{\infty}\left(0, \infty ; V \cap H^{2}(\Omega)\right)
$$

We introduce the energy of problem (1.1)-(1.7) as

$$
E(t)=\frac{1}{2}\left\|u^{\prime}\right\|^{2}+\frac{1}{2} a(u, u)+\frac{h}{2}\left\|\nabla u^{\prime}\right\|^{2}+\frac{1}{4}\|\Delta v\|^{2} .
$$

Now, we are ready to state the following main result.

Theorem 2.1 Assume that $(\mathrm{H} 1)$ and $(\mathrm{H} 2)$ hold. Suppose that $D$ is a positive $C^{1}$ function, with $D(0)=0$, for which $H_{0}$ is a strictly increasing and strictly convex $C^{2}$ function on $(0, r]$ and

$$
\int_{0}^{+\infty} \frac{g(s)}{H_{0}^{-1}\left(-g^{\prime}(s)\right)} d s<+\infty
$$

Then there exist positive constants $k_{1}, k_{2}, k_{3}$ and $\epsilon_{0}$ such that the solution of (1.1)-(1.7) satisfies

$$
E(t) \leq k_{3} H_{1}^{-1}\left(k_{1} t+k_{2}\right), \quad \forall t \geq 0,
$$

where

$$
H_{1}(t)=\int_{t}^{1} \frac{1}{s H_{0}^{\prime}\left(\epsilon_{0} s\right)} d s \quad \text { and } \quad H_{0}(t)=H(D(t)) .
$$

Moreover, for some choice of $D$, if $\int_{0}^{1} H_{1}(t) d t<+\infty$, then we obtain

$$
E(t) \leq k_{3} G^{-1}\left(k_{1} t+k_{2}\right)
$$

where

$$
G(t)=\int_{t}^{1} \frac{1}{s H^{\prime}\left(\epsilon_{0} s\right)} d s
$$

In particular, (2.11) is valid for the special case $H(t)=c t^{p}$ for $1 \leq p<\frac{3}{2}$.

Remark 2.1 If $F$ is a convex function on $[a, b], f: \Omega \rightarrow[a, b]$ and $h$ are integrable functions on $\Omega, h(x) \geq 0$, and $\int_{\Omega} h(x) d x=h_{0}>0$, then Jensen's inequality states that

$$
F\left(\frac{1}{h_{0}} \int_{\Omega} f(x) h(x) d x\right) \leq \frac{1}{h_{0}} \int_{\Omega} F(f(x)) h(x) d x .
$$


Remark 2.2 1. From the properties of $H$, we can show that the function $H_{1}$ is strictly decreasing and convex on $(0,1]$, with $\lim _{t \rightarrow 0} H_{1}(t)=+\infty$. Then Theorem 2.1 ensures

$$
\lim _{t \rightarrow+\infty} E(t)=0
$$

2. By using (H1) and (H2), we conclude that $\lim _{t \rightarrow+\infty} g(t)=0$. This implies that $\lim _{t \rightarrow+\infty}\left(-g^{\prime}(t)\right)$ cannot be equal to a positive number, and so it is natural to assume that $\lim _{t \rightarrow+\infty}\left(-g^{\prime}(t)\right)=0$. Therefore, there is $t_{0}>0$ large enough such that $g\left(t_{0}\right)>0$ and

$$
\max \left\{g(t),-g^{\prime}(t)\right\}<\min \left\{r, H(r), H_{0}(r)\right\}, \quad \forall t \geq t_{0} .
$$

Since $g$ is nonincreasing, $g(0)>0$ and $g\left(t_{0}\right)>0$, we obtain

$$
0<g\left(t_{0}\right) \leq g(t) \leq g(0), \quad \forall t \in\left[0, t_{0}\right]
$$

From $H$ is a positive continuous function, we have

$$
c_{1} \leq H(g(t)) \leq c_{2}, \quad \forall t \in\left[0, t_{0}\right]
$$

for some positive constants $c_{1}$ and $c_{2}$. Hence, by (2.4), (2.15) and (2.16),

$$
g^{\prime}(t) \leq-H(g(t)) \leq-\frac{c_{1}}{g(0)} g(0) \leq-\frac{c_{1}}{g(0)} g(t)
$$

which gives

$$
g^{\prime}(t) \leq-c_{3} g(t), \quad \forall t \in\left[0, t_{0}\right]
$$

for some positive constant $c_{3}$.

\section{General decay of the energy}

In this section we prove the decay rates in Theorem 2.1. The following result shows the dissipative property of system (1.1)-(1.7). Multiplying (1.1) by $u^{\prime}(t)$, we have the identity

$$
E^{\prime}(t)=a\left(g * u, u^{\prime}\right)
$$

We define the modified energy by

$$
\mathcal{E}(t)=\frac{1}{2}\left\|u^{\prime}\right\|^{2}+\frac{h}{2}\left\|\nabla u^{\prime}\right\|^{2}+\frac{1}{2}\left(1-\int_{0}^{t} g(s) d s\right) a(u, u)+\frac{1}{2} g \square \partial^{2} u+\frac{1}{4}\|\Delta v\|^{2} .
$$

From (2.5) and (3.1), we get

$$
\mathcal{E}^{\prime}(t)=-\frac{1}{2} g(t) a(u, u)+\frac{1}{2} g^{\prime} \square \partial^{2} u .
$$

This implies that $\mathcal{E}(t)$ is nonincreasing, and from (2.3) one sees that

$$
E(t) \leq \frac{1}{1-l} \mathcal{E}(t), \quad \forall t \geq 0
$$


First, let us define the perturbed modified energy by

$$
L(t)=N \mathcal{E}(t)+\epsilon \Phi(t)+\Psi(t)
$$

where

$$
\Phi(t)=\int_{\Omega} u^{\prime} u d \Omega+h \int_{\Omega} \nabla u^{\prime} \nabla u d \Omega
$$

and

$$
\Psi(t)=\int_{\Omega}\left(h \Delta u^{\prime}-u^{\prime}\right) \int_{0}^{t} g(t-s)(u(t)-u(s)) d s d \Omega
$$

Using the ideas presented in [9], we easily obtain the following lemmas.

Lemma 3.1 For $N>0$ large enough, there exist $\alpha_{1}>0$ and $\alpha_{2}>0$ such that

$$
\alpha_{1} \mathcal{E}(t) \leq L(t) \leq \alpha_{2} \mathcal{E}(t), \quad \forall t \geq 0
$$

Proof By Young's inequality, (2.2) and (2.3), we have

$$
|\Phi(t)| \leq \frac{1}{2}\left\|u^{\prime}\right\|^{2}+\frac{h}{2}\left\|\nabla u^{\prime}\right\|^{2}+\frac{C_{p}+C_{s} h}{2} a(u, u)
$$

and

$$
|\Psi(t)| \leq \frac{1}{2}\left\|u^{\prime}\right\|^{2}+\frac{h}{2}\left\|\nabla u^{\prime}\right\|^{2}+\frac{\left(C_{p}+C_{s} h\right) l}{2} g \square \partial^{2} u
$$

From (3.6) and (3.7) we obtain

$$
\begin{aligned}
|L(t)-N \mathcal{E}(t)| \leq & \frac{1}{2}(\epsilon+1)\left\|u^{\prime}\right\|^{2}+\frac{h}{2}(\epsilon+1)\left\|\nabla u^{\prime}\right\|^{2} \\
& +\frac{\left(C_{p}+C_{s} h\right) \epsilon}{2} a(u, u)+\frac{\left(C_{p}+C_{s} h\right) l}{2} g \square \partial^{2} u \\
\leq & C_{0} \mathcal{E}(t),
\end{aligned}
$$

where $C_{0}$ is a positive constant depending on $\epsilon, h, C_{p}, C_{s}$ and $l$. Choosing $N>0$ large, we complete the proof of Lemma 3.1.

Lemma 3.2 For each $t_{0}>0$ and sufficiently large $N>0$, there exist positive constants $\beta_{1}$ and $\beta_{2}$ such that

$$
L^{\prime}(t) \leq-\beta_{1} \mathcal{E}(t)+\beta_{2} g \square \partial^{2} u, \quad \forall t \geq t_{0}
$$

Proof Direct computations, using (1.1), yield

$$
\Phi^{\prime}(t)=-a(u, u)+a(g * u, u)+([u, v], u)+\left\|u^{\prime}\right\|^{2}+h\left\|\nabla u^{\prime}\right\|^{2}
$$


By Young's inequality, we have

$$
a(g * u, u) \leq(\delta+1)\left(\int_{0}^{t} g(s) d s\right) a(u, u)+\frac{1}{4 \delta} g \square \partial^{2} u
$$

From (3.9) and (3.10), we get

$$
\begin{aligned}
\Phi^{\prime}(t) \leq & \left\|u^{\prime}\right\|^{2}+h\left\|\nabla u^{\prime}\right\|^{2}+\frac{1}{4 \delta} g \square \partial^{2} u \\
& -\left(1-(\delta+1) \int_{0}^{t} g(s) d s\right) a(u, u)-\|\Delta v\|^{2},
\end{aligned}
$$

where $\delta>0$. Similarly we deduce

$$
\begin{aligned}
\Psi^{\prime}(t)= & \int_{0}^{t} g(t-s) a(u(t)-u(s), u(t)) d s-\int_{0}^{t} g(t-s)(u(t)-u(s),[u, v]) d s \\
& -\int_{0}^{t} g(t-s) a\left(u(t)-u(s), \int_{0}^{t} g(t-\tau) u(\tau) d \tau\right) d s \\
& -h \int_{0}^{t} g^{\prime}(t-s)\left(\nabla u(t)-\nabla u(s), \nabla u^{\prime}(t)\right) d s-h\left(\int_{0}^{t} g(s) d s\right)\left\|\nabla u^{\prime}\right\|^{2} \\
& -\int_{0}^{t} g^{\prime}(t-s)\left(u(t)-u(s), u^{\prime}(t)\right) d s-\left(\int_{0}^{t} g(s) d s\right)\left\|u^{\prime}\right\|^{2} \\
:= & I_{1}+I_{2}+\cdots+I_{5}-h\left(\int_{0}^{t} g(s) d s\right)\left\|\nabla u^{\prime}\right\|^{2}-\left(\int_{0}^{t} g(s) d s\right)\left\|u^{\prime}\right\|^{2} .
\end{aligned}
$$

Now, we estimate the terms on the right-hand side of (3.12). Since $E(t)$ is bounded, we have that $\|v\|_{\infty} \leq \int_{\Omega}|\Delta v|^{2} d \Omega$ is also bounded, and then Young's and Hölder's inequalities, (2.2), (2.3), (2.6) and (2.7) give that

$$
\begin{aligned}
\left|I_{1}\right| & \leq \eta\left(\int_{0}^{t} g(s) d s\right) a(u, u)+\frac{1}{4 \eta} g \square \partial^{2} u, \\
\left|I_{2}\right| & =\int_{\Omega}\left[u, \int_{0}^{t} g(t-s)(u(t)-u(s)) d s\right] v d \Omega \leq \eta a(u, u)+c_{\eta} l\|v\|_{\infty}^{2} g \square \partial^{2} u, \\
\left|I_{3}\right| & \leq l \int_{0}^{t} g(t-s) a(u(t)-u(s), u(t)-u(s)) d s+l \int_{0}^{t} g(t-s) a(u(t)-u(s), u(t)) d s \\
& \leq\left(l+\frac{l}{4 \eta}\right) g \square \partial^{2} u+l^{2} \eta a(u, u), \\
\left|I_{4}\right| & \leq h \eta\left\|\nabla u^{\prime}\right\|^{2}+\frac{h}{4 \eta} \int_{\Omega}\left(\int_{0}^{t} g^{\prime}(t-s)|\nabla u(t)-\nabla u(s)| d s\right)^{2} d \Omega \\
& \leq h \eta\left\|\nabla u^{\prime}\right\|^{2}-\frac{g(0) C_{s} h}{4 \eta} g^{\prime} \square \partial^{2} u, \\
\left|I_{5}\right| & \leq \eta\left\|u^{\prime}\right\|^{2}+\frac{1}{4 \eta} \int_{\Omega}\left(\int_{0}^{t} g^{\prime}(t-s)|u(t)-u(s)| d s\right)^{2} d \Omega \\
& \leq \eta\left\|u^{\prime}\right\|^{2}-\frac{g(0) C_{p}}{4 \eta} g^{\prime} \square \partial^{2} u .
\end{aligned}
$$


From the above estimates, we see that

$$
\begin{aligned}
\Psi^{\prime}(t) \leq & \left(h \eta-h \int_{0}^{t} g(s) d s\right)\left\|\nabla u^{\prime}\right\|^{2}+\left(\eta-\int_{0}^{t} g(s) d s\right)\left\|u^{\prime}\right\|^{2}+\eta\left(1+l+l^{2}\right) a(u, u) \\
& +\left(l+\frac{l}{4 \eta}+\frac{1}{4 \eta}+c_{\eta} l\|v\|_{\infty}^{2}\right) g \square \partial^{2} u-\frac{g(0)\left(C_{s} h+C_{p}\right)}{4 \eta} g^{\prime} \square \partial^{2} u .
\end{aligned}
$$

Let $\int_{0}^{t_{0}} g(s) d s:=g_{0}$, where $t_{0}$ was introduced in (2.13). Since $g$ is positive, we have $\int_{0}^{t} g(s) d s \geq g_{0}$ for all $t \geq t_{0}$. Thus, making use of this and combining (3.2), (3.4), (3.11) and (3.13), we obtain

$$
\begin{aligned}
L^{\prime}(t) \leq & -\left(g_{0}-\eta-\epsilon\right)\left\|u^{\prime}\right\|^{2}-h\left(g_{0}-\eta-\epsilon\right)\left\|\nabla u^{\prime}\right\|^{2} \\
& -\left[\frac{N}{2} g(t)+\epsilon(1-(1+\delta) l)-\eta\left(1+l+l^{2}\right)\right] a(u, u) \\
& -\epsilon\|\Delta v\|^{2}+\left(\frac{N}{2}-\frac{g(0)\left(C_{s} h+C_{p}\right)}{4 \eta}\right) g^{\prime} \square \partial^{2} u \\
& +\left(l+\frac{\epsilon}{4 \delta}+\frac{1}{4 \eta}+\frac{l}{4 \eta}+c_{\eta} l\|v\|_{\infty}^{2}\right) g \square \partial^{2} u .
\end{aligned}
$$

We first take $\epsilon>0$ and $\delta>0$ so small that $g_{0}-\epsilon>0$ and $1-(1+\delta) l>0$, respectively. And then, we choose $\eta>0$ sufficiently small so that $g_{0}-\eta-\epsilon>0$ and $\epsilon(1-(1+\delta) l)-\eta(1+l+$ $\left.l^{2}\right)>0$. Finally, taking $N>0$ large enough, we deduce that (3.8).

Proof of Theorem 2.1 From (2.17), (3.2) and (3.8), we have

$$
\begin{aligned}
L^{\prime}(t) \leq & -\beta_{1} \mathcal{E}(t)-\frac{\beta_{2}}{c_{3}} \int_{0}^{t_{0}} g^{\prime}(t-s) a(u(t)-u(s), u(t)-u(s)) d s \\
& +\beta_{2} \int_{t_{0}}^{t} g(t-s) a(u(t)-u(s), u(t)-u(s)) d s \\
\leq & -\beta_{1} \mathcal{E}(t)-\frac{2 \beta_{2}}{c_{3}} \mathcal{E}^{\prime}(t)+\beta_{2} \int_{t_{0}}^{t} g(t-s) a(u(t)-u(s), u(t)-u(s)) d s .
\end{aligned}
$$

We take $\mathcal{L}(t)=L(t)+\frac{2 \beta_{2}}{c_{3}} \mathcal{E}(t)$, which is clearly equivalent to $\mathcal{E}(t)$. By (3.14), we get, for all $t \geq t_{0}$,

$$
\mathcal{L}^{\prime}(t) \leq-\beta_{1} \mathcal{E}(t)+\beta_{2} \int_{t_{0}}^{t} g(s) a(u(t)-u(t-s), u(t)-u(t-s)) d s
$$

(A) The special case $H(t)=c t^{p}$ and $1 \leq p<\frac{3}{2}$.

Case 1. $p=1$. Using (2.4) and (3.2), estimate (3.15) yields

$$
\begin{aligned}
\mathcal{L}^{\prime}(t) & \leq-\beta_{1} \mathcal{E}(t)-\frac{\beta_{2}}{c} \int_{t_{0}}^{t} g^{\prime}(s) a(u(t)-u(t-s), u(t)-u(t-s)) d s \\
& \leq-\beta_{1} \mathcal{E}(t)-\frac{2 \beta_{2}}{c} \mathcal{E}^{\prime}(t),
\end{aligned}
$$


which gives

$$
\left(\mathcal{L}+\frac{2 \beta_{2}}{c} \mathcal{E}\right)^{\prime}(t) \leq-\beta_{1} \mathcal{E}(t), \quad \forall t \geq t_{0}
$$

From (3.3) and (3.5), we see that $\mathcal{L}+\frac{2 \beta_{2}}{c} \mathcal{E} \sim \mathcal{E} \sim E$. Then we have

$$
E(t) \leq c^{\prime} e^{-c t}=c^{\prime} G^{-1}(t)
$$

where

$$
G(t)=\int_{t}^{1} \frac{1}{s H^{\prime}\left(\epsilon_{0} s\right)} d s=\int_{t}^{1} \frac{1}{s c} d s=-\frac{\ln t}{c} .
$$

Case $2.1<p<\frac{3}{2}$. By (2.4) we obtain

$$
g^{\prime}(t) \leq-c g^{p}(t), \quad 1<p<\frac{3}{2} .
$$

Using (2.3) and (3.17) we see that

$$
\int_{0}^{\infty} g^{1-\theta}(s) d s<\infty
$$

for any $\theta<2-p$. By (3.2) and (3.18) and taking $t_{0}$ even larger if needed, we deduce that, for all $t \geq t_{0}$,

$$
\begin{aligned}
k(t) & :=\int_{t_{0}}^{t} g^{1-\theta}(s) a(u(t)-u(t-s), u(t)-u(t-s)) d s \\
& \leq 2 \int_{t_{0}}^{t} g^{1-\theta}(s)[a(u(t), u(t))+a(u(t-s), u(t-s))] d s \\
& \leq c \mathcal{E}(0) \int_{t_{0}}^{t} g^{1-\theta}(s) d s<1 .
\end{aligned}
$$

From Hölder's inequality, Jensen's inequality (2.13), (3.2), (3.17) and (3.19), we have

$$
\begin{aligned}
& \int_{t_{0}}^{t} g(s) a(u(t)-u(t-s), u(t)-u(t-s)) d s \\
& =\int_{t_{0}}^{t} g^{(p-1+\theta)\left(\frac{\theta}{p-1+\theta}\right)}(s) g^{1-\theta}(s) a(u(t)-u(t-s), u(t)-u(t-s)) d s \\
& \quad \leq\left(\int_{t_{0}}^{t} g^{p-1+\theta}(s) g^{1-\theta}(s) a(u(t)-u(t-s), u(t)-u(t-s)) d s\right)^{\frac{\theta}{p-1+\theta}} \\
& \quad \times\left(\int_{t_{0}}^{t} g^{1-\theta}(s) a(u(t)-u(t-s), u(t)-u(t-s)) d s\right)^{\frac{p-1}{p-1+\theta}} \\
& =k(t)\left(\frac{1}{k(t)} \int_{t_{0}}^{t} g^{p-1+\theta}(s) g^{1-\theta}(s) a(u(t)-u(t-s), u(t)-u(t-s)) d s\right)^{\frac{\theta}{p-1+\theta}}
\end{aligned}
$$




$$
\begin{aligned}
& \leq\left(\int_{t_{0}}^{t} g^{p}(s) a(u(t)-u(t-s), u(t)-u(t-s)) d s\right)^{\frac{\theta}{p-1+\theta}} \\
& \leq\left(\frac{1}{c}\right)^{\frac{\theta}{p-1+\theta}}\left(\int_{t_{0}}^{t}-g^{\prime}(s) a(u(t)-u(t-s), u(t)-u(t-s)) d s\right)^{\frac{\theta}{p-1+\theta}} \\
& \leq\left(\frac{1}{c}\right)^{\frac{\theta}{p-1+\theta}}\left(-\mathcal{E}^{\prime}(t)\right)^{\frac{\theta}{p-1+\theta}} .
\end{aligned}
$$

Then, using (3.20), we show that (3.15) yields, for $\theta=\frac{1}{2}$,

$$
\mathcal{L}^{\prime}(t) \leq-\beta_{1} \mathcal{E}(t)+\frac{\beta_{2}}{c^{\frac{1}{2 p-1}}}\left(-\mathcal{E}^{\prime}(t)\right)^{\frac{1}{2 p-1}}
$$

Multiplying (3.21) by $\mathcal{E}^{\gamma}(t)$, with $\gamma=2 p-2$, and using (3.2) and Young's inequality, we obtain

$$
\begin{aligned}
\left(\mathcal{L} \mathcal{E}^{\gamma}\right)^{\prime}(t) & =\mathcal{L}^{\prime}(t) \mathcal{E}^{\gamma}(t)+\gamma \mathcal{L}(t) \mathcal{E}^{\gamma-1}(t) \mathcal{E}^{\prime}(t) \leq-\beta_{1} \mathcal{E}^{\gamma+1}(t)+\frac{\beta_{2}}{c^{\frac{1}{\gamma+1}}} \mathcal{E}^{\gamma}(t)\left(-\mathcal{E}^{\prime}(t)\right)^{\frac{1}{\gamma+1}} \\
& \leq-\beta_{1} \mathcal{E}^{\gamma+1}(t)+\varepsilon \mathcal{E}^{\gamma+1}(t)+C_{\varepsilon}\left(-\mathcal{E}^{\prime}(t)\right)
\end{aligned}
$$

Taking $\varepsilon<\beta_{1}$, we have, for some $C_{1}>0$,

$$
L_{0}^{\prime}(t) \leq-C_{1} L_{0}^{\gamma+1}(t)
$$

where $L_{0}=\mathcal{L E}^{\gamma}+C_{\varepsilon} \mathcal{E} \sim \mathcal{E} \sim E$. Therefore we deduce that

$$
E(t) \leq \frac{c}{\left(c^{\prime}+c^{\prime \prime} t\right)^{\frac{1}{\gamma}}} .
$$

Since $p<\frac{3}{2}$ and by (3.22), we find that

$$
\int_{0}^{\infty} E(t) d t \leq \int_{0}^{\infty} \frac{c}{\left(c^{\prime}+c^{\prime \prime} t\right)^{\frac{1}{2 p-2}}} d t<+\infty
$$

Using this fact, we have

$$
\int_{0}^{t} a(u(t)-u(t-s), u(t)-u(t-s)) d s \leq c \int_{0}^{t} E(s) d s<+\infty .
$$

Hence, from (3.2), (3.17), (3.23) and Hölder's inequality, estimate (3.15) gives

$$
\begin{aligned}
\mathcal{L}^{\prime}(t) & \leq-\beta_{1} \mathcal{E}(t)+\beta_{2}\left(\int_{0}^{t} a(u(t)-u(t-s), u(t)-u(t-s)) d s\right)^{\frac{p-1}{p}}\left(g^{p} \square \partial^{2} u\right)^{\frac{1}{p}} \\
& \leq-\beta_{1} \mathcal{E}(t)+c\left(-g^{\prime} \square \partial^{2} u\right)^{\frac{1}{p}} \leq-\beta_{1} \mathcal{E}(t)+c\left(-\mathcal{E}^{\prime}(t)\right)^{\frac{1}{p}} .
\end{aligned}
$$

Now, we multiply (3.24) by $\mathcal{E}^{\gamma}(t)$, with $\gamma=p-1$. Then, repeating the above steps, we see that

$$
E(t) \leq \frac{c}{\left(c^{\prime}+c^{\prime \prime} t\right)^{\frac{1}{\gamma}}}=c G^{-1}\left(a^{\prime}+a^{\prime \prime} t\right)
$$


where

$$
G(t)=\frac{1}{c p \epsilon_{0}^{p-1}} \int_{t}^{1} \frac{1}{s^{p}} d s=\frac{1}{c p(p-1) \epsilon_{0}^{p-1}}\left(\frac{1}{t^{p-1}}-1\right) .
$$

(B) The general case. This case is obtained on account of the ideas presented in [24, 25] as follows. Let $H_{0}^{*}$ be the convex conjugate of $H_{0}$ in the sense of Young (see [29]); then

$$
H_{0}^{*}(s)=s\left(H_{0}^{\prime}\right)^{-1}(s)-H_{0}\left[\left(H_{0}^{\prime}\right)^{-1}(s)\right], \quad \text { if } s \in\left(0, H_{0}^{\prime}(r)\right]
$$

and $H_{0}^{*}$ satisfies the following Young's inequality:

$$
A B \leq H_{0}^{*}(A)+H_{0}(B), \quad \text { if } A \in\left(0, H_{0}^{\prime}(r)\right], B \in(0, r] .
$$

We define $\eta(t)$ by

$$
\eta(t):=\int_{t_{0}}^{t} \frac{g(s)}{H_{0}^{-1}\left(-g^{\prime}(s)\right)} a(u(t)-u(t-s), u(t)-u(t-s)) d s,
$$

where $H_{0}$ is such that (2.9) is satisfied. As in (3.19), we find that $\eta(t)$ satisfies

$$
\eta(t)<1, \quad \forall t \geq t_{0} .
$$

Furthermore, we define $I(t)$ by

$$
I(t):=-\int_{t_{0}}^{t} g^{\prime}(s) \frac{g(s)}{H_{0}^{-1}\left(-g^{\prime}(s)\right)} a(u(t)-u(t-s), u(t)-u(t-s)) d s .
$$

Since $H_{0}$ is strictly convex on $(0, r]$ and $H_{0}(0)=0$, then

$$
H_{0}(\lambda x) \leq \lambda H_{0}(x)
$$

provided $0 \leq \lambda \leq 1$ and $x \in(0, r]$. From (3.28), (3.29) and Jensen's inequality (2.13), we obtain

$$
\begin{aligned}
I(t) & =\frac{1}{\eta(t)} \int_{t_{0}}^{t} \eta(t) H_{0}\left[H_{0}^{-1}\left(-g^{\prime}(s)\right)\right] \frac{g(s)}{H_{0}^{-1}\left(-g^{\prime}(s)\right)} a(u(t)-u(t-s), u(t)-u(t-s)) d s \\
& \geq \frac{1}{\eta(t)} \int_{t_{0}}^{t} H_{0}\left[\eta(t) H_{0}^{-1}\left(-g^{\prime}(s)\right)\right] \frac{g(s)}{H_{0}^{-1}\left(-g^{\prime}(s)\right)} a(u(t)-u(t-s), u(t)-u(t-s)) d s \\
& \geq H_{0}\left(\int_{t_{0}}^{t} g(s) a(u(t)-u(t-s), u(t)-u(t-s)) d s\right) .
\end{aligned}
$$

This implies that

$$
\int_{t_{0}}^{t} g(s) a(u(t)-u(t-s), u(t)-u(t-s)) d s \leq H_{0}^{-1}(I(t)) .
$$

Using (3.15) and (3.30) we see that

$$
\mathcal{L}^{\prime}(t) \leq-\beta_{1} \mathcal{E}(t)+\beta_{2} H_{0}^{-1}(I(t)), \quad \forall t \geq t_{0} .
$$


By (2.3), (2.4) and the properties of $H_{0}$ and $D$, we have

$$
\frac{g(s)}{H_{0}^{-1}\left(-g^{\prime}(s)\right)} \leq \frac{g(s)}{H_{0}^{-1}(H(g(s)))}=\frac{g(s)}{D^{-1}(g(s))} \leq \delta_{0}
$$

for some positive constant $\delta_{0}$. Thus, using (2.13), (3.2) and (3.32) and choosing $t_{0}$ even larger, we can find that $I(t)$ satisfies, for all $t \geq t_{0}$,

$$
\begin{aligned}
I(t) & \leq-\delta_{0} \int_{t_{0}}^{t} g^{\prime}(s) a(u(t)-u(t-s), u(t)-u(t-s)) d s \leq-c \mathcal{E}(0) \int_{t_{0}}^{t} g^{\prime}(s) d s \\
& \leq c g\left(t_{0}\right) \mathcal{E}(0) \leq \min \left\{r, H(r), H_{0}(r)\right\} .
\end{aligned}
$$

Now, for $\epsilon_{0}<r$ and $d_{0}>0$, we define the functional

$$
F_{1}(t):=\mathcal{L}(t) H_{0}^{\prime}\left(\epsilon_{0} \frac{\mathcal{E}(t)}{\mathcal{E}(0)}\right)+d_{0} \mathcal{E}(t)
$$

which satisfies

$$
d_{1} F_{1}(t) \leq \mathcal{E}(t) \leq d_{2} F_{1}(t)
$$

for some $d_{1}, d_{2}>0$. From (3.33), we have $H_{0}^{-1}(I(t)) \leq r$. Also, by $\epsilon_{0}<r, \mathcal{E}^{\prime} \leq 0$, we get $\epsilon_{0} \frac{\mathcal{E}(t)}{\mathcal{E}(0)}<r$. Using the fact that $\mathcal{E}^{\prime} \leq 0, H_{0}>0, H_{0}^{\prime}>0$ and $H_{0}^{\prime \prime}>0$ on $(0, r]$ and (3.2), (3.26), (3.27), (3.31) and (3.33), we obtain

$$
\begin{aligned}
F_{1}^{\prime}(t) \leq & -\beta_{1} \mathcal{E}(t) H_{0}^{\prime}\left(\epsilon_{0} \frac{\mathcal{E}(t)}{\mathcal{E}(0)}\right)+\beta_{2} H_{0}^{\prime}\left(\epsilon_{0} \frac{\mathcal{E}(t)}{\mathcal{E}(0)}\right) H_{0}^{-1}(I(t))+d_{0} \mathcal{E}^{\prime}(t) \\
\leq & -\beta_{1} \mathcal{E}(t) H_{0}^{\prime}\left(\epsilon_{0} \frac{\mathcal{E}(t)}{\mathcal{E}(0)}\right)+\beta_{2} H_{0}^{*}\left(H_{0}^{\prime}\left(\epsilon_{0} \frac{\mathcal{E}(t)}{\mathcal{E}(0)}\right)\right)+\beta_{2} I(t)+d_{0} \mathcal{E}^{\prime}(t) \\
\leq & -\beta_{1} \mathcal{E}(t) H_{0}^{\prime}\left(\epsilon_{0} \frac{\mathcal{E}(t)}{\mathcal{E}(0)}\right)+\beta_{2} \epsilon_{0} \frac{\mathcal{E}(t)}{\mathcal{E}(0)} H_{0}^{\prime}\left(\epsilon_{0} \frac{\mathcal{E}(t)}{\mathcal{E}(0)}\right) \\
& -\beta_{2} H_{0}\left(\epsilon_{0} \frac{\mathcal{E}(t)}{\mathcal{E}(0)}\right)+\beta_{2} I(t)+d_{0} \mathcal{E}^{\prime}(t) \\
\leq & -\left(\beta_{1} \mathcal{E}(0)-\beta_{2} \epsilon_{0}\right) \frac{\mathcal{E}(t)}{\mathcal{E}(0)} H_{0}^{\prime}\left(\epsilon_{0} \frac{\mathcal{E}(t)}{\mathcal{E}(0)}\right)-2 \beta_{2} \delta_{0} \mathcal{E}^{\prime}(t)+d_{0} \mathcal{E}^{\prime}(t)
\end{aligned}
$$

Therefore, with a suitable choice of $\epsilon_{0}$ and $d_{0}$, we see that

$$
F_{1}^{\prime}(t) \leq-k\left(\frac{\mathcal{E}(t)}{\mathcal{E}(0)}\right) H_{0}^{\prime}\left(\epsilon_{0} \frac{\mathcal{E}(t)}{\mathcal{E}(0)}\right)=-k H_{2}\left(\frac{\mathcal{E}(t)}{\mathcal{E}(0)}\right), \quad \forall t \geq t_{0},
$$

where $k>0$ and $H_{2}(t)=t H_{0}^{\prime}\left(\epsilon_{0} t\right)$. From the strict convexity of $H_{0}$ on $(0, r]$, we find that $H_{2}(t)>0$ and $H_{2}^{\prime}(t)=H_{0}^{\prime}\left(\epsilon_{0} t\right)+\epsilon_{0} t H_{0}^{\prime \prime}\left(\epsilon_{0} t\right)>0$ on $(0,1]$. We take

$$
R(t)=\frac{d_{1} F_{1}(t)}{\mathcal{E}(0)}
$$

which is clearly equivalent to $\mathcal{E}(t)$. By (3.34), (3.35) and $H_{2}^{\prime}>0$, we have

$$
R^{\prime}(t) \leq-\frac{k d_{1}}{\mathcal{E}(0)} H_{2}\left(\frac{\mathcal{E}(t)}{\mathcal{E}(0)}\right) \leq-k_{0} H_{2}(R(t)), \quad \forall t \geq t_{0},
$$


where $k_{0}=\frac{k d_{1}}{\mathcal{E}(0)}>0$. Hence, a simple integration gives, for some $k_{1}, k_{2}>0$,

$$
R(t) \leq H_{1}^{-1}\left(k_{1} t+k_{2}\right), \quad \forall t \geq t_{0}
$$

where $H_{1}(t)=\int_{t}^{1} \frac{1}{H_{2}(s)} d s$. Here, we have used, on the basis of the properties of $H_{2}$, the fact that $H_{1}$ is a strictly decreasing function on $(0,1]$ and $\lim _{t \rightarrow 0} H_{1}(t)=+\infty$. From (3.3), (3.34) and (3.36), estimate (2.10) is established.

Moreover, if $\int_{0}^{t} H_{1}(t) d t<+\infty$, then

$$
\int_{0}^{t} a(u(t)-u(t-s), u(t)-u(t-s)) d s \leq c \int_{0}^{t} E(s) d s<+\infty
$$

Similarly, we define, for large $t_{0}$,

$$
\eta(t):=\int_{t_{0}}^{t} a(u(t)-u(t-s), u(t)-u(t-s)) d s<1
$$

and

$$
I(t):=-\int_{t_{0}}^{t} g^{\prime}(s) a(u(t)-u(t-s), u(t)-u(t-s)) d s
$$

Using (2.4), the strict convexity of $H$ and Jensen's inequality (2.13), we have

$$
\begin{aligned}
I(t) & \geq \frac{1}{\eta(t)} \int_{t_{0}}^{t} \eta(t) H(g(s)) a(u(t)-u(t-s), u(t)-u(t-s)) d s \\
& \geq \frac{1}{\eta(t)} \int_{t_{0}}^{t} H(\eta(t) g(s)) a(u(t)-u(t-s), u(t)-u(t-s)) d s \\
& \geq H\left(\frac{1}{\eta(t)} \int_{t_{0}}^{t} \eta(t) g(s) a(u(t)-u(t-s), u(t)-u(t-s)) d s\right) \\
& =H\left(\int_{t_{0}}^{t} g(s) a(u(t)-u(t-s), u(t)-u(t-s)) d s\right) .
\end{aligned}
$$

Thus, we deduce that

$$
\int_{t_{0}}^{t} g(s) a(u(t)-u(t-s), u(t)-u(t-s)) d s \leq H^{-1}(I(t))
$$

and (3.15) becomes

$$
\mathcal{L}^{\prime}(t) \leq-\beta_{1} \mathcal{E}(t)+\beta_{2} H^{-1}(I(t)), \quad \forall t \geq t_{0}
$$

Therefore, repeating the same procedures, we find that for some $k_{1}, k_{2}$ and $k_{3}>0$,

$$
E(t) \leq k_{3} G^{-1}\left(k_{1} t+k_{2}\right)
$$

where $G(t)=\int_{t}^{1} \frac{1}{s H^{\prime}\left(\epsilon_{0} s\right)} d s$. 
Example We give an example to illustrate the energy decay rates given by Theorem 2.1.

If

$$
g(t)=\frac{1}{a+t^{q}}
$$

for $q>3$ and $a>1$ chosen so that $g$ satisfies (2.3), then $g^{\prime}(t)=-H(g(t))$, where

$$
H(t)=q t^{2}\left(\frac{1}{t}-a\right)^{1-\frac{1}{q}}
$$

Since

$$
H^{\prime}(t)=\frac{q\left(1+\frac{1}{q}-2 a t\right)}{\left(\frac{1}{t}-a\right)^{\frac{1}{q}}}, \quad H^{\prime \prime}(t)=\frac{\frac{2 a^{2} q}{t^{2}}\left(t-\frac{1+q-\sqrt{q^{2}-1}}{2 a q}\right)\left(t-\frac{1+q+\sqrt{q^{2}-1}}{2 a q}\right)}{\left(\frac{1}{t}-a\right)^{1+\frac{1}{q}}},
$$

then the function $H$ satisfies hypothesis (H2) on the interval $(0, r]$ for any $0<r<\frac{1+q-\sqrt{q^{2}-1}}{2 a q}$. By choosing $D(t)=t^{\alpha},(2.9)$ is satisfied for any $\alpha>\frac{q}{q-1}$. Then an explicit rate of decay can be obtained by Theorem 2.1. The function $H_{0}(t)=H\left(t^{\alpha}\right)$ has derivative

$$
H_{0}^{\prime}(t)=\frac{q \alpha t^{\alpha-1}\left[1+\frac{1}{q}-2 a t^{\alpha}\right]}{\left(\frac{1}{t^{\alpha}}-a\right)^{\frac{1}{q}}} .
$$

Hence,

$$
H_{1}(t)=\int_{t}^{1} \frac{\left[\frac{1}{\left(\epsilon_{0} s\right)^{\alpha}}-a\right]^{\frac{1}{q}}}{q \alpha s\left(\epsilon_{0} s\right)^{\alpha-1}\left[1+\frac{1}{q}-2 a\left(\epsilon_{0} s\right)^{\alpha}\right]} d s .
$$

Let $\frac{1}{\left(\epsilon_{0} s\right)^{\alpha}}=u$, then we have

$$
H_{1}(t)=\int_{\frac{1}{\epsilon_{0}^{\alpha}}}^{\frac{1}{\left(\epsilon_{0} t\right)^{\alpha}}} \frac{(u-a)^{\frac{1}{q}} u^{-\frac{1}{\alpha}}}{q \alpha^{2}\left[1+\frac{1}{q}-\frac{2 a}{u}\right]} d u \leq \frac{1}{q \alpha^{2}\left[1+\frac{1}{q}-2 a \epsilon_{0}^{\alpha}\right]} \int_{\frac{1}{\epsilon_{0}^{\alpha}}}^{\frac{1}{\left(\epsilon_{0} t\right)^{\alpha}}}(u-a)^{\frac{1}{q}} u^{-\frac{1}{\alpha}} d u .
$$

Using the fact that the function $f(u)=(u-a)^{\frac{1}{q}}$ is increasing on $(a,+\infty)$ and $(u-a)^{\frac{1}{q}}<u^{\frac{1}{q}}$ and taking $\epsilon_{0}<a^{-\frac{1}{\alpha}}$, then

$$
H_{1}(t) \leq \frac{1}{q \alpha^{2}\left[1+\frac{1}{q}-2 a \epsilon_{0}^{\alpha}\right]} \int_{\frac{1}{\epsilon_{0}^{\alpha}}}^{\frac{1}{\left(\epsilon_{0} t\right)^{\alpha}}} u^{\frac{1}{q}-\frac{1}{\alpha}} d u=\frac{\epsilon_{0}^{\frac{q-\alpha-\alpha q}{q}}}{\alpha(\alpha-q+\alpha q)\left[1+\frac{1}{q}-2 a \epsilon_{0}^{\alpha}\right]}\left[t^{\frac{q-\alpha-\alpha q}{q}}-1\right] .
$$

Now, we find that if $\alpha<\frac{2 q}{1+q}$,

$$
\begin{aligned}
\int_{0}^{1} H_{1}(t) d t & \leq \frac{\epsilon_{0}^{\frac{q-\alpha-\alpha q}{q}}}{\alpha(\alpha-q+\alpha q)\left[1+\frac{1}{q}-2 a \epsilon_{0}^{\alpha}\right]} \int_{0}^{1}\left[t^{\frac{q-\alpha-\alpha q}{q}}-1\right] d t \\
& =\frac{\epsilon_{0}^{\frac{q-\alpha-\alpha q}{q}}}{\alpha(2 q-\alpha-\alpha q)\left[1+\frac{1}{q}-2 a \epsilon_{0}^{\alpha}\right]}<+\infty .
\end{aligned}
$$


Choosing $\frac{1}{\epsilon_{0} s}=v$ and $\epsilon_{0}<a^{-1}$, we obtain

$$
\begin{aligned}
G(t) & =\int_{t}^{1} \frac{1}{s H^{\prime}\left(\epsilon_{0} s\right)} d s=\int_{t}^{1} \frac{\left(\frac{1}{\epsilon_{0} s}-a\right)^{\frac{1}{q}}}{s q\left(1+\frac{1}{q}-2 a \epsilon_{0} s\right)} d s=\int_{\frac{1}{\epsilon_{0}}}^{\frac{1}{\epsilon_{0} t}} \frac{(v-a)^{\frac{1}{q}} v^{-1}}{q\left(1+\frac{1}{q}-\frac{2 a}{v}\right)} d v \\
& \leq \frac{1}{q\left(1+\frac{1}{q}-2 a \epsilon_{0}\right)} \int_{\frac{1}{\epsilon_{0}}}^{\frac{1}{\epsilon_{0} t}} v^{\frac{1}{q}-1} d v=\frac{1}{1+\frac{1}{q}-2 a \epsilon_{0}}\left[\left(\frac{1}{\epsilon_{0} t}\right)^{\frac{1}{q}}-\left(\frac{1}{\epsilon_{0}}\right)^{\frac{1}{q}}\right]
\end{aligned}
$$

Therefore,

$$
G^{-1}(t) \leq \frac{1}{\epsilon_{0}\left[\left(\frac{1}{\epsilon_{0}}\right)^{\frac{1}{q}}+\left(1+\frac{1}{q}-2 a \epsilon_{0}\right) t\right]^{q}}
$$

Then we can use (2.11) to deduce that the energy decays at the same rate of $g$, that is,

$$
E(t) \leq \frac{\tilde{c}_{1}}{\tilde{c}_{2}+\tilde{c}_{3} t^{q}}
$$

where $\tilde{c}_{i}(i=1,2,3)$ are constants.

\section{Competing interests}

The author declares that she has no competing interests.

\section{Author's contributions}

The work was realized by the author.

\section{Acknowledgements}

This work was supported by the Dong-A University research fund

Received: 7 August 2015 Accepted: 27 October 2015 Published online: 05 November 2015

\section{References}

1. Favini, A, Horn, M, Lasiecka, I, Tataru, D: Global existence, uniqueness and regularity of solutions to a von Kármán system with nonlinear boundary dissipation. Differ. Integral Equ. 9(2), 267-294 (1996)

2. Horn, MA, Lasiecka, I: Uniform decay of weak solutions to a von Kármán plate with nonlinear boundary dissipation. Differ. Integral Equ. 7, 885-908 (1994)

3. Horn, MA, Lasiecka, I: Global stabilization of a dynamic von Kármán plate with nonlinear boundary feedback. Appl. Math. Optim. 31, 57-84 (1995)

4. Puel, J, Tucsnak, M: Boundary stabilization for the von Kármán equations. SIAM J. Control 33, 255-273 (1996)

5. Rivera, JEM, Menzala, GP: Decay rates of solutions to a von Kármán system for viscoelastic plates with memory. Q. Appl. Math. LVII, 181-200 (1999)

6. Rivera, JEM, Oquendo, HP, Santos, ML: Asymptotic behavior to a von Kármán plate with boundary memory conditions. Nonlinear Anal. 62, 1183-1205 (2005)

7. Santos, ML, Soufyane, A: General decay to a von Kármán plate system with memory boundary conditions. Differ. Integral Equ. 24(1-2), 69-81 (2011)

8. Raposo, CA, Santos, ML: General decay to a von Kármán system with memory. Nonlinear Anal. 74, 937-945 (2011)

9. Kang, JR: Energy decay rates for von Kármán system with memory and boundary feedback. Appl. Math. Comput. 218, 9085-9094 (2012)

10. Cavalcanti, MM, Domingos Cavalcanti, VN, Ferreira, J: Existence and uniform decay for a non-linear viscoelastic equation with strong damping. Math. Methods Appl. Sci. 24, 1043-1053 (2001)

11. Cavalcanti, MM, Domingos Cavalcanti, VN, Prates Filho, JA, Soriano, JA: Existence and uniform decay rates for viscoelastic problems with nonlinear boundary damping. Differ. Integral Equ. 14(1), 85-116 (2001)

12. Cavalcanti, MM, Domingos Cavalcanti, VN, Soriano, JA: Exponential decay for the solution of semilinear viscoelastic wave equation with localized damping. Electron. J. Differ. Equ. 2002, 44 (2002)

13. Han, XS, Wang, MX: Global existence and uniform decay for a nonlinear viscoelastic equation with damping. Nonlinear Anal. 70, 3090-3098 (2009)

14. Berrimi, S, Messaoudi, SA: Existence and decay of solutions of a viscoelastic equation with a nonlinear source. Nonlinear Anal. 64, 2314-2331 (2006)

15. Messaoudi, SA, Tatar, NE: Exponential and polynomial decay for a quasilinear viscoelastic equation. Nonlinear Anal. 68, 785-793 (2008)

16. Liu, WJ: Uniform decay of solutions for a quasilinear system of viscoelastic equations. Nonlinear Anal. 71, 2257-2267 (2009) 
17. Messaoudi, SA: General decay of solutions of a viscoelastic equation. J. Math. Anal. Appl. 341, 1457-1467 (2008)

18. Han, XS, Wang, MX: General decay of energy for a viscoelastic equation with nonlinear damping. Math. Methods Appl. Sci. 32, 346-358 (2009)

19. Guesmia, A, Messaoudi, SA: General energy decay estimates of Timoshenko systems with frictional versus viscoelastic damping. Math. Methods Appl. Sci. 32, 2102-2122 (2009)

20. Ferreira, J, Messaoudi, SA: On the general decay of a nonlinear viscoelastic plate equation with a strong damping and $\vec{p}(x, t)$-Laplacian. Nonlinear Anal. 104, 40-49 (2014)

21. Messaoudi, SA: General decay of the solution energy in a viscoelastic equation with a nonlinear source. Nonlinear Anal. 69, 2589-2598 (2008)

22. Messaoudi, SA, Mustafa, Ml: On convexity for energy decay rates of a viscoelastic equation with boundary feedback. Nonlinear Anal. 72, 3602-3611 (2010)

23. Park, JY, Park, SH: Decay rate estimates for wave equations of memory type with acoustic boundary conditions. Nonlinear Anal. 74, 993-998 (2011)

24. Mustafa, MI, Messaoudi, SA: General stability result for viscoelastic wave equations. J. Math. Phys. 53, 053702 (2012)

25. Messaoudi, SA, Mustafa, Ml: A general stability result for a quasilinear wave equation with memory. Nonlinear Anal., Real World Appl. 14, 1854-1864 (2013)

26. Alabau-Boussouira, F, Cannarsa, P: A general method for proving sharp energy decay rates for memory dissipative evolution equations. C. R. Acad. Sci. Paris, Ser. I 347, 867-872 (2009)

27. Cavalcanti, MM, Cavalcanti, ADD, Lasiecka, I, Wang, X: Existence and sharp decay rate estimates for a von Karman system with long memory. Nonlinear Anal., Real World Appl. 22, 289-306 (2015)

28. Lagnese, J: Boundary Stabilization of Thin Plates. SIAM, Philadelphia (1989)

29. Arnold, VI: Mathematical Methods of Classical Mechanics. Springer, New York (1989)

\section{Submit your manuscript to a SpringerOpen ${ }^{\circ}$ journal and benefit from:}

- Convenient online submission

Rigorous peer review

- Immediate publication on acceptance

- Open access: articles freely available online

- High visibility within the field

- Retaining the copyright to your article 